\title{
Using COTS Technologies for Battlefield Applications
}

\author{
Anni Woolley \\ Department of the Army \\ CERDEC C2D \\ Aberdeen Proving Ground, MD 21001 USA
}

\author{
Danielle Duff \\ Department of the Army \\ CERDEC C2D \\ Aberdeen Proving Ground, MD 21001 USA
}

\begin{abstract}
Microsoft Office is the most widely used non Program of Record Mission Command system. Warfighters have been somewhat successful in using Commercial Off-The Shelf software (COTS) to meet their needs, but truly integrating COTS software with Army systems can often be problematic. Some COTS products, such as Microsoft Office, and Microsoft's Office Communications Server (OCS) are designed to be extended. Such extensibility suggests that this type of COTS product can be more tightly integrated with existing Army systems. CERDEC's Collaborative Battlespace Reasoning and Awareness Army Technology Objective (COBRA ATO) has as a central goal to research and develop ways to enhance collaboration among Warfighters. One of the main methods to achieve this goal is to integrate familiar COTS tools with Army Battle Command System (ABCS) applications. This paper describes several different integration approaches which include integrating Excellike spread sheet capabilities into the Army Command Post of the Future (CPOF); bringing the Common Operational Picture (COP) into PowerPoint; researching Open Data protocol and its application; integrating OCS with Force XXI Battle Command Brigade and Below (FBCB2), Single Channel Ground \& Airborne Radio System (SINCGARS) and other commonly used communication end points in the field. This paper presents the technical approaches, lessons learned and path forward for the efforts.
\end{abstract}

Keywords - OCS, Lync, FBCB2, SINCGARS, CPOF, Microsoft Office, $U C B$

\section{INTRODUCTION}

The Collaborative Battlespace Reasoning and Awareness Army Technology Objective (COBRA ATO) is a four year (FY9-FY12) research effort lead by the Command and Control Directorate (C2D). The main focus of the COBRA is to explore technologies that can help enhance the collaboration and data sharing between the operations (OPS) and Intelligence (INTEL) officers and staff. In addition to these major product lines, COBRA also provides various studies in support of unanticipated needs as may be required by its customers and senior leaders. One of the major product lines that COBRA is specifically researching is how to integrate Microsoft products with Army systems. Microsoft product integration is crucial because these products are readily available and widely used across all military domains. Furthermore, Microsoft products tend to be highly developed tools that seamlessly integrate with each other. Training is minimal because most military personnel are already familiar with these products. Even though Microsoft products are widely used, they often do not easily integrate with military systems. A lack of integration can force the introduction of repetitive, tedious, and error-prone tasks on the battlefield. For example, data elements may be stored in a spreadsheet yet also need to be entered into a collaborative system, such as CPOF to provide COP or Situational Awareness (SA) information. Currently, a user would need to type the information into both the spreadsheet and CPOF. To avoid this extra work, the Microsoft Integration objective attempts to integrate familiar and available COTS tools with ABCS applications. The eventual goal is to ease Warfighters' tasks and better utilize the already significant Army investment on Microsoft and Government Off The Shelf (GOTS) products.

This paper describes the inspiration for the Microsoft Integration efforts and provides several examples of work that is being done by COBRA. The main focus of this paper is on the discussion of the Office Communications Server (OCS) effort and its demonstration at Army Team Command, Control, Communications, Computers, Intelligence Surveillance, Reconnaissance (C4ISR) On The Move (OTM) Event 10 (E10) at Ft. Dix, NJ.

This paper is organized as follows: Section II discusses the approaches used to achieve the objective for the Microsoft integration initiative. Section III describes the OCS effort. Section IV presents the OCS effort technical details. Section V discusses the advantages and disadvantages of OCS. Section VI provides conclusions and suggestions for further research.

\section{TECHNICAL APPROACHES}

The objective for the Microsoft integration initiative is to integrate familiar Microsoft products with ABCS applications. To achieve this objective the following three approaches were identified.

\section{A. Adding Tactical capabilities to COTS products}

One approach is to add tactical capabilities to COTS products where it makes sense. For example, putting a COP into PowerPoint was identified under this approach. The majority of military systems come pre-installed with PowerPoint because Microsoft Office is part of the Army Golden Master [1], but not nearly as many systems have the CPOF client. Therefore, if the COP from CPOF could be integrated into PowerPoint, users without CPOF clients could 
collaborate and share the same COP via PowerPoint. The Microsoft Integration team was able to prototype this capability successfully using CPOF Mapboarding, XMPP server and PowerPoint 2007.

\section{B. Enhancing GOTS to work seamlessly with COTS products}

The next approach is to enhance GOTS products with COTS capabilities. An example of this approach is adding COTS spreadsheets capability into CPOF. There is much information captured in the battlefield by using Microsoft Excel. In order to share the information with others and perform mission planning steps on CPOF, the operators had to retype the information from one application to another. The work is tedious and error prone. The opportunity to assist the warfighters was clear: Add a spreadsheet capability to CPOF. A spreadsheet stickie with basic spreadsheet functionality, such as copy, paste, cut, formula, etc., was developed. The capability was implemented, transitioned to PM Mission Command (PM $\mathrm{MC})$, and included in the CPOF BC 13 release. Figure 1 shows an instance of the spreadsheet stickie in CPOF.

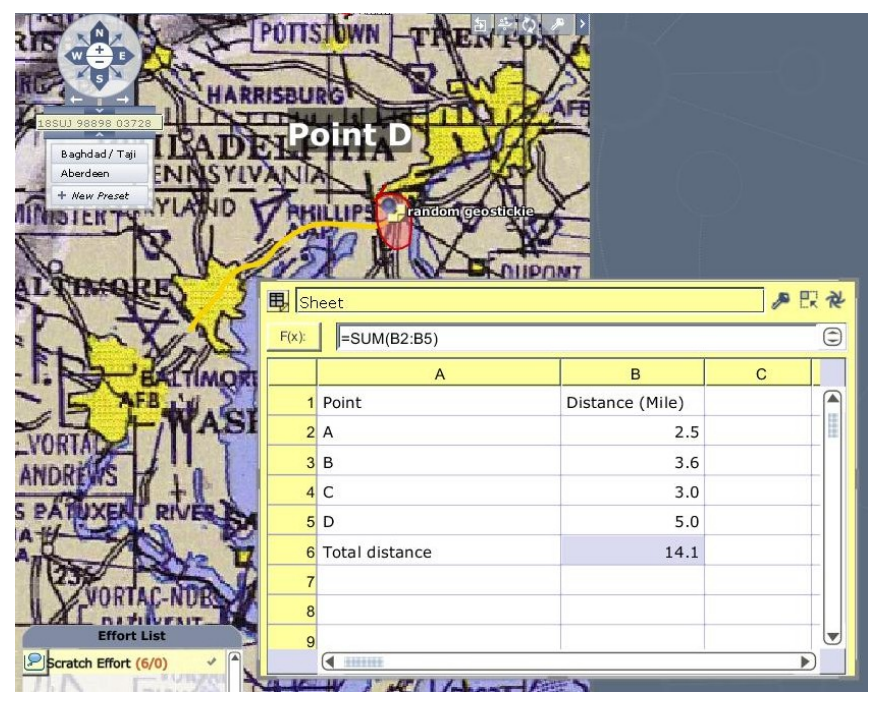

Figure 1 Spreadsheet Stickie Enhances CPOF

\section{Researching emerging COTS technologies and their applicability in the military domain}

The final approach focuses on researching emerging COTS technologies and their applicability in the military domain. The Open Data Protocol (OData) and OCS technologies were both identified as interesting potential candidates.

OData shows promise for easing the application developers' task of exposing and accessing information from a variety of sources via the web. It is an open standard web protocol for querying and updating data that provides a way to unlock data and free it from the silos created by some applications [2]. A CPOF OData Provider is currently under development that exposes CPOF data via OData. The provider will ease the task of accessing/updating CPOF data without requiring knowledge of the CPOF $3^{\text {rd }}$ Party Development Kit (3PDK).

OCS was identified as a research target for several reasons. The Army owns licenses for this product, but it is underutilized. Also, a need for a common suite of tools that enables multi-form collaboration at the lower echelon is identified in the U.S. Army Training and Doctrine Command (TRADOC) Mission Command Essential Capabilities (MCEC) [3]. Consequently, there is interest in knowing more about the capabilities of OCS and in determining if OCS could possibly fill the technology gap. The OCS research effort will be discussed further in the subsequent sections.

\section{OCS DEMONSTRATION AT C4ISR ON THE Move E10 AT FORT DIX, NJ}

As part of the Microsoft Integration effort, COBRA is exploring the enterprise and tactical capabilities of OCS. OCS is Microsoft's solution for providing unified communications. The Army owns OCS 2007 R1 licenses, but they are restricted. Obtaining the software requires an approval process. The C4ISR OTM E10 demonstration was created using Microsoft OCS 2007 R2 Enterprise. It should be noted that all capabilities discussed in this paper could have been implemented with the OCS 2007 R1 version. The team's objective was to create a concept demonstration for C4ISR OTM E10 at Ft. Dix to see if military communications could be enhanced by using COTS products and if those COTS solutions could be integrated with negligible change to the existing communications infrastructure currently in use in the field. Specifically, the demonstration shows how OCS can be leveraged in a tactical environment.

To make OCS relevant in a military environment, COBRA examined current analog and Voice Over IP (VoIP) telephony, web and video conferencing, chat, email, and radio systems that make up the military communications network. The analog and VoIP Telephony and web conferencing integration was straight forward since it falls in line with commercial integrations with OCS (See section IV.A).

Text-based chat, however, is a more difficult issue because there are many incompatible chat systems, such as Variable Message Format (VMF), Internet Relay Chat (mIRC), etc., used in a tactical environment. Since this demonstration was conceptual, the demonstration concentrated on one typical chat scenario, (VMF messages to OCS) in order to show chat feasibility in a tactical environment. OCS accepts Extensible Messaging and Presence Protocol (XMPP) chat messages through an OCS XMPP Gateway, but many of the typical military chat systems are not based on the XMPP, so this creates compatibility issues. To bridge this gap, COBRA developed Universal Collaboration Bridge (UCB), was leveraged to provide interoperability. Details regarding this solution are described in section IV.B.

Collaboration with radio users is another important area of interest in a military environment, so radio integration was also investigated. To examine the relevance of radio communications, the SINCGARS was chosen since it is commonly used and was readily available for this experiment. Custom hardware and software were developed to integrate tactical and commercial radios into the experiment. Detailed information regarding the radio integration solution is outlined in section IV.C. 


\section{TECHNICAL DETAIL FOR OCS IMPLEMENTATION}

To introduce OCS into a relevant military testing environment, $\mathrm{ABCS}$ and tactically relevant COTS solutions were integrated with OCS to form the communications network. In particular, the SINCGARS, FBCB2, and an open source Private Branch Exchange (PBX) were included in the demonstration to represent an existing telecommunication network. Figure 2 is a network diagram that shows the radios, servers, and other equipment that composed the demonstration architecture.

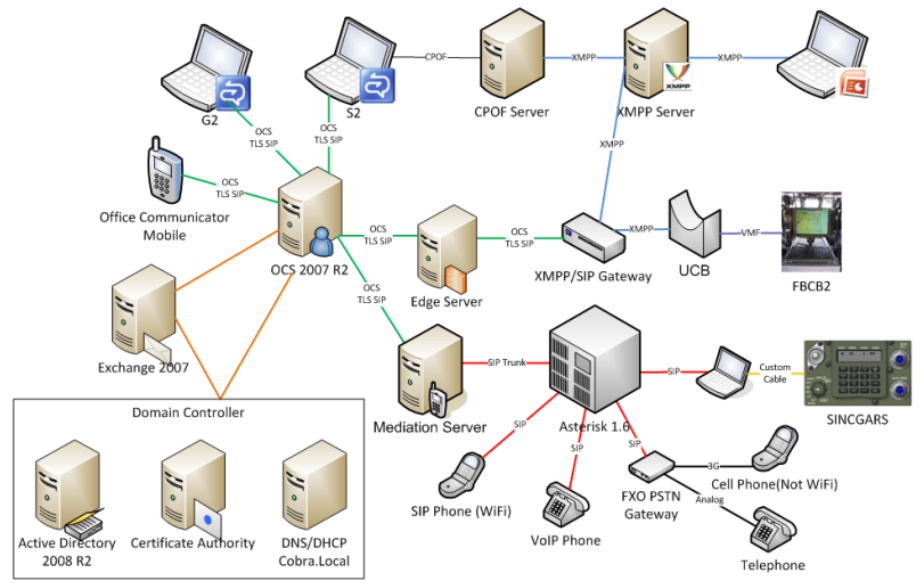

Figure 2 OCS Demonstration Network Diagram

\section{A. Private Branch Exchange (PBX) Integration}

To connect to existing telecommunication systems, the OCS Mediation Server functioned as an interface between OCS and PBX. For the C4ISR OTM E10 demonstration, PBX represented an existing communications network composed with various communications media, including VoIP Phone, WiFi Phone, SINCGARS, and Commercial Radios. Furthermore, the communication network also included a Public Switched Telephone Network (PSTN), which connected to PBX via the Foreign Exchange Office (FXO) Gateway.

\section{B. Text-Based Chat Integration}

Microsoft provides the OCS XMPP gateway which enables IM and presence communications between OCS and XMPPbased IM and presence providers. Out of box OCS could enable chat between OCS client and Jabber, Google Talk, etc. However, it would not work with many of the non-XMPP based military chat/message systems. For example, FBCB2 and Blue Force Tracker are based on VMF. To bridge this gap, the UCB was leveraged to enable collaboration between the various chat systems. This product was inserted into the network architecture to enable interoperability among collections of different types of text-based chat systems. UCB is designed to be an extensible collaboration interoperability framework, which enables interoperability among collections of different types of text-based chat systems. Each chat standard can be inserted in the UCB as a plug-in. It could allow OCS clients to chat with others using XMPP, VMF, IRC, etc. XMPP and VMF plug-ins were utilized for this experiment. This allows the FBCB2/Blue Force Tracker Operator to send VMF messages, such as spot report, to an OCS user. They could also communicate back and forth via free text message and chat. Figure 3 shows the interactions.

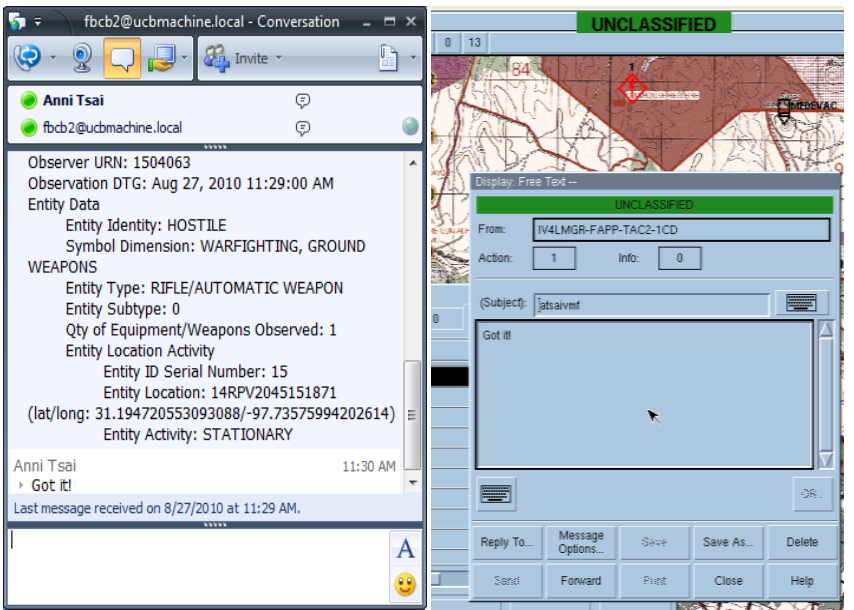

Figure 3 Interactions between OCS client and FBCB2

\section{Radio Integration}

An in house project was initiated to develop an appropriate USB to radio interface using commercial hardware along with a software implementation of push to talk (PTT) based on Conaito SIP Stack. For commercial radios, we purchased a USB-radio interface. For SINCGARS, a custom cable (See Figure 4) was built to connect SINCGARS to COTS USB Radio Interface (URI) (See Figure 5). With this technology, a radio acting as a base station can be connected to a laptop which is accessible in the IP network. Other radios communicating within the base station's radio net can then be reached via the OCS client and other telephony devices.
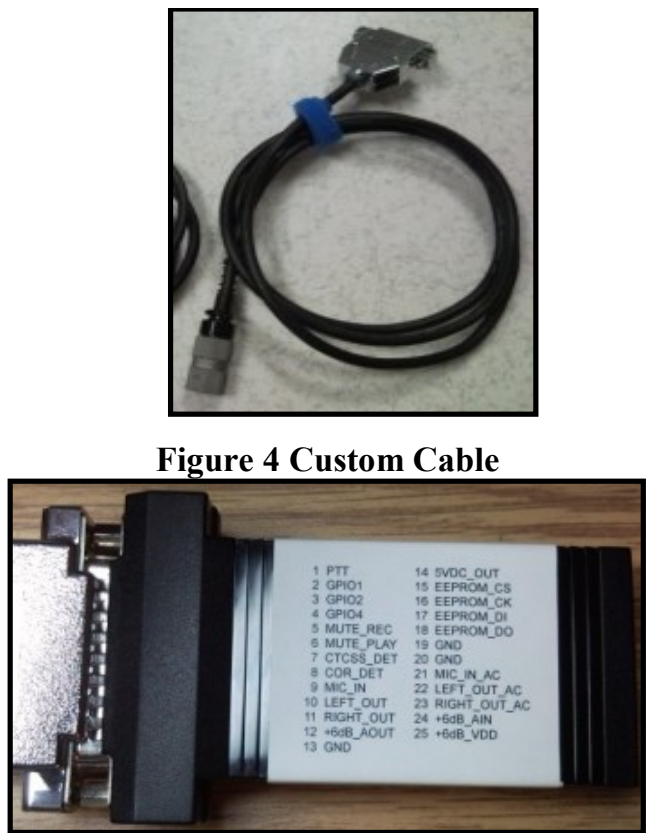

Figure 5 DMK URI

\section{ADVANTAGES AND DISADVANTAGES OF OCS}

After setting up and evaluating OCS in the laboratory, several advantages and disadvantages became readily apparent. 


\section{A. OCS Advantages}

OCS streamlines the communications process for users. Even though rigorous analysis on the communications speed and the ease of use of OCS has not been performed, various advantages of using OCS are easily observed.

- Users no longer need to look up phone numbers to make a voice call.

- Users have more options on the types of media, such as mobile phone, radio, VoIP phone, PSTN phone, email, IM, etc., to use.

- Fewer steps are required to set up a new type of communication (one click and you can call, IM, email, share video, join conference, etc.).

- $\quad$ Since OCS is a software solution, the communications can easily be implemented into business workflow.

- OCS has an extensible Software Development Kit (SDK), which allows developers to embed OCS functionality into existing applications.

- OCS integrates well with other Microsoft Office Products, such as Outlook, Word, etc. For example, a user can see presence information in Word and start the communication right in the Word document. See Figure 6.

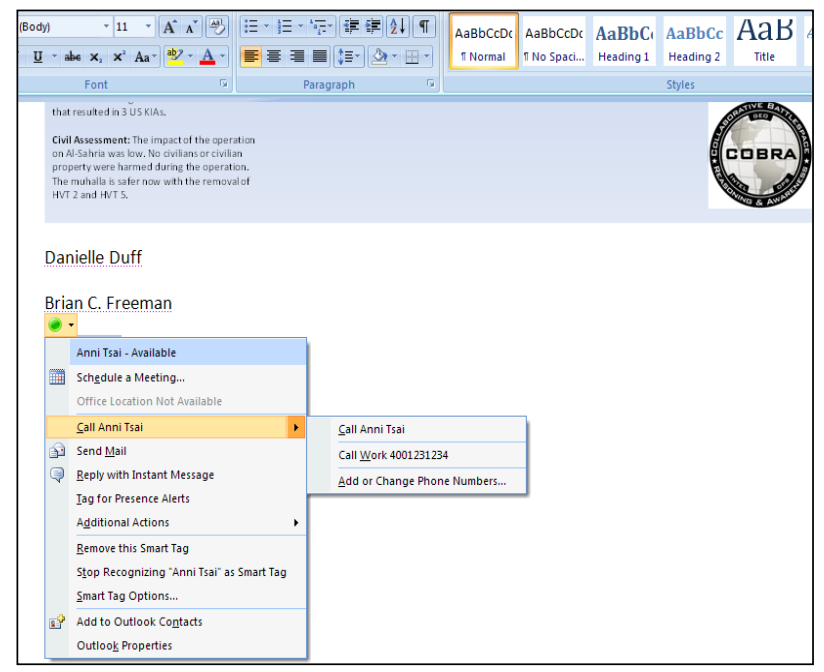

Figure 6 OCS Implementation in Word

\section{B. OCS Disadvantages}

The military environment is more complex and may require additional code to integrate with older and less secure technologies. In any complex hardware and software environment, clear and complete examinations must take place prior to the addition of more software into the environment. The following is a subset of issues that need to be addressed:

\section{- Security}

- Although OCS uses secure Session Initiation Protocol (SIP) communications requirements, security will need to be examined when integrating with older and less secure technologies.

- Sustainability and Maintainability

- $\quad$ The additional code required for integration could introduce new support costs.

- The addition of servers increases infrastructure and complexity on the Mission Command Stacks.

\section{CONCLUSIONS AND FUTURE EFFORTS}

AT C4ISR OTM E10, the COBRA ATO team was able to create an environment to demonstrate the viability of OCS in a military setting using existing communications infrastructure. Current telephony (analog and VOIP phones) as well as cell phones were able to interoperate with OCS. Calls initiated from a Microsoft Office user were streamlined, since the user no longer needed to know which phone number was best to reach the intended recipient or dial the number. The creation of conference calls was demonstrated and it was noted that these calls required far fewer steps than using the current Enterprise solution Defense Connect Online (DCO), mainly due to the fact that OCS integrates seamlessly with Microsoft Office products. Both chat and radio were successfully demonstrated by adding software designed specifically for the tactical domain problems and integrating these solutions into the OCS architecture. Integration of the military chat environment was demonstrated through the bridging of VMF and OCS chat messages. The architecture used to show chat collaboration can be easily extended to the other chat systems used on the battlefield such as mIRC. OCS and SINCGARS radio integration was demonstrated with a minimal set of software and hardware. The ease at which an OCS client user could make a call to a radio without a prior knowledge of the user's communication medium was also noted.

The last capability tested at C4ISR OTM E10 was presence. Being able to access presence information inside a variety of Microsoft Office products also adds to the ease of collaboration. For example, while reading over a report, it was shown that you could see if the author was online and initiates a collaboration session with that person from within Microsoft Word. Technology convenience such as the previous example directly shows that OCS would definitely make a positive impact in the battlefield as well as in the Army Enterprise.

In conclusion, COBRA was able to create a concept demonstration at OTM E10 that shows how tactical and Enterprise collaboration can be enhanced using OCS. While no metrics were collected in regard to increase in productivity, etc., dramatic improvements in collaboration were observed. Since collaboration is the one of the most important tools in the decision making process, it seems to be valuable to continue this research. Additionally, there are many advantages of utilizing COTS solutions, such as reducing life cycle costs. Since most of people are familiar with Microsoft Office, the training cost can be reduced as well. Furthermore, the Army already owns Microsoft Office and OCS 2007 licenses, so finding solutions to better utilize the products makes sense. Since soldiers are using COTS, especially Microsoft Office, the 
more we can adapt and adopt these tools, the less expensive it will be to field capabilities sooner.

At present, progress has been made in several areas. US Army owns a limited number of OCS 2007 licenses, therefore it was important to see if this technology could be used with the current software set available. The team installed it and validated that the integrated capabilities still persist. In order to keep up with the emerging capabilities, the team also upgraded OCS to its latest version, Microsoft Lync 2010, and tested against it. Therefore, C2D has verified that the conceptual integration effort mentioned in this paper can work on all three of these versions.

In the future, COBRA ATO team will be continuing research in a variety of avenues. First, the team will begin to research the value of integrating OCS/Lync presence and communications functionality into GOTs products, such as CPOF. Additionally, improvements to radio integration will be made, such as bi-directional calling. Furthermore, C2D will investigate the operating environment for the battlefield applications being deployed. C2D has verified the bandwidth requirements for OCS published by Microsoft shown in Table 1 [4]. From the surface, OCS/Lync is an enterprise solution and can be easily be targeted for higher echelon. The question C2D will try to answer is can we utilize this solution at a lower

\section{Table 1 OCS Bandwidth Requirements}

\begin{tabular}{|l|l|l|}
\hline Bandwidth Requirements & Required & Recommended \\
\hline Data & $56 \mathrm{kbps}$ & $56 \mathrm{kbps}$ \\
\hline Voice & $50 \mathrm{kbps}$ & $80 \mathrm{kbps}$ \\
\hline Video & $50 \mathrm{kbps}$ & $350 \mathrm{kbps}$ \\
\hline Microsoft RoundTable & $50 \mathrm{kbps}$ & $350 \mathrm{kbps}$ \\
\hline
\end{tabular}

echelon and where it should be deployed within existing tactical architecture.

The COBRA Microsoft integration team will continue to explore the use of OCS and/or Microsoft Lync as a collaboration solution for the battlefield, and investigate other relevant integration efforts between COTS and GOTS. The team will be exploring transition opportunities with PM MC, PM Warfighter Information Network-Tactical (WIN-T) and PEO Integration and will be reaching out for collaborative opportunities such as leveraging research in the business platform community and exploration into radio communications domain.

\section{ACKNOWLEDGMENT}

Thanks to the entire COBRA team for making this work possible.

\section{REFERENCES}

[1] Army Golden Master. https://www.us.army.mil/suite/page/130061

[2] Open Data Protocol (OData). http://www.odata.org/

[3] The Mission Command Essential Capabilities (MCEC) White Paper, 19 June 2010.

[4] Office Communications Server 2007 Evaluation Guide. 